\title{
Article
}

\section{Optimal polynomial decay for a coupled system of wave with past history}

\author{
S. M. S. Cordeiro' ${ }^{1}$, R. F. C. Lobato ${ }^{1}$ and C. A. Raposo ${ }^{2, *}$ \\ 1 Faculty of Exact Sciences and Technology Federal University of Pará 68440-000, Abaetetuba, PA, Brazil. \\ 2 Federal University of São João del-Rey and PhD Program of the Federal University of Bahia 40170-110, Salvador, BA, \\ Brazil. \\ * Correspondence: hakemali@yahoo.com
}

Received: 5 February 2020; Accepted: 8 April 2020; Published: 10 April 2020.

\begin{abstract}
This work deals with a coupled system of wave with past history effective just in one of the equations. We show that the dissipation given by the memory effect is not strong enough to produce exponential decay. On the other hand, we show that the solution of this system decays polynomially with rate $t^{-\frac{1}{2}}$. Moreover by recent result due to A. Borichev and Y. Tomilov, we show that the rate is optimal. To the best of our knowledge, there is no result for optimal rate of polynomial decay for coupled wave systems with memory in the previous literature.
\end{abstract}

Keywords: Coupled system of waves equation, polynomial decay, memory, optimality.

MSC: Primary 35b40; Secondary 35L51, 35A01.

\section{Introduction}

I

$\mathrm{n}$ this paper we consider a coupled system of wave with past history given by

$$
\begin{aligned}
& u_{t t}-\Delta u+\int_{0}^{\infty} g(s) \Delta u(t-s) d s+\alpha v=0 \text { in } \Omega \times(0, \infty), \\
& v_{t t}-\Delta v+\alpha u=0 \quad \text { in } \Omega \times(0, \infty) \\
& u=v=0 \text { on } \Gamma \times(0, \infty), \\
& (u(x, 0), v(x, 0))=\left(u_{0}(x), v_{0}(x)\right), \text { in } \Omega, \\
& \left(u_{t}(x, 0), v_{t}(x, 0)\right)=\left(u_{1}(x), v_{1}(x)\right), \quad \text { in } \Omega,
\end{aligned}
$$

where $\Omega$ is an open bounded set of $\mathbb{R}^{n}$ with smooth boundary $\Gamma$.

The above model can be used to describe the evolution of a system consisting of two elastic membranes subject to an elastic force that attracts one membrane to the other with coefficient $\alpha>0$. Note that the term $\int_{0}^{\infty} g(s) \Delta u(t-s) d s$, acts on the first membrane as a stabilizer.

Many interesting physical phenomena such as viscoelasticity, hereditary polarization in dielectrics, population dynamics or heat flow in real conductors, to name some, are modeled by differential equations which are influenced by the past values of one or more variables in play so-called equations with memory. The main problem in the analysis of equations of this kind lies in their nonlocal character, due to the presence of the memory term given by the time convolution of the unknown function against a suitable memory kernel. The memory term may can produce loss of exponential stability for the system, [1]. The history of nonlocal problems with integral conditions for partial differential equations is recent and goes back to [2]. In [3], a review of the progress in the nonlocal models with integral type was given with many discussions related to physical justifications, advantages, and numerical applications.

Coupled wave system has been considered in various contexts. In [4] both wave equations are damped on the boundary and the coupling is effected by compact operator and exponential stability is obtained when the boundary damping is linear. Boundary damping is also considered in $[5,6]$. On exact boundary controllability for linearly coupled wave equations, we refer [7]. Uniform exponential stability was given 
in [8] for wave equations coupled in parallel with coupling distributed springs and viscous dampers due to different boundary conditions and wave propagation speeds.

For weak damping acting only one equation, the optimal polynomial decay to coupled wave equations was studied in [9]. In [10], it was proved that the energy of associated coupled system weakly dissipative decays polynomially with explicit polynomial decay rates for sufficiently smooth solutions. In [11], under new compatibility assumptions, the authors proved polynomial decay for the energy of solutions and optimized previous results by interpolation techniques introduced in [10].

On the asymptotic behavior of the coupled system (1)-(5) we refer the work [12] where the authors proved by method introduced in [11] that the solution has a polynomial rate of decay. The central question of this work is to analyze what is the best decay rate of the system (1)-(5). In this direction, we prove that the associated semigroup decays with rate $t^{-\frac{1}{2}}$. Moreover we show that the rate is optimal. For what we know in the literature the optimal rate of polynomial decay for coupled wave systems with memory was not previously considered.

The mathematical structure of the paper is organized as follows: In Section 2 we discuss the existence, regularity and uniqueness of strong solutions of the system (1)-(5) by semigroup technique, see [13]. In Section 3 we study the lack of exponential decay using Prüss's results [14]. Finally in section 4 we show that the system is polynomially stable giving an optimal decay rate. That is, this rate cannot be improved. For this we use the recent result due to Borichev and Tomilov [15].

\section{Semigroup Setup}

Following the approach of Dafermos [16] and Fabrizio and Morro [17], we consider $\eta=\eta^{t}(s)$, the relative history of $u$, defined as

$$
\eta=\eta^{t}(s)=u(t)-u(t-s)
$$

Hence, putting

$$
\beta_{0}=1-\int_{0}^{\infty} g(s) d s>0
$$

the system (1)-(5) turns into the system

$$
\begin{aligned}
& u_{t t}-\beta_{0} \Delta u-\int_{0}^{\infty} g(\tau) \Delta \eta(\cdot, \tau) d \tau+\alpha v=0 \quad \text { in } \Omega \times(0, \infty), \\
& v_{t t}-\Delta v+\alpha u=0 \quad \text { in } \Omega \times(0, \infty), \\
& \eta_{t}+\eta_{s}-u_{t}=0, \quad \text { in } \Omega \times(0, \infty) \\
& u=v=\eta^{t}(s)=0 \quad \text { on } \Gamma \times(0, \infty), \forall s \geq 0 \\
& (u(x, 0), v(x, 0))=\left(u_{0}(x), v_{0}(x)\right) \text { in } \Omega, \\
& \left(u_{t}(x, 0), v_{t}(x, 0)=\left(u_{1}(x), v_{1}(x)\right) \text { in } \Omega,\right. \\
& \eta_{0}(\cdot, s)=u_{0}(\cdot, 0)-u_{0}(\cdot,-s), \quad \Omega \times(\infty),
\end{aligned}
$$

where the third equation is obtained differentiating (6) with respect to $s$ and the condition (13) means that the history is considered as an initial value.

We study the existence and uniqueness of solutions for the system (7)-(13) using the semigroup techniques. As in [18], we use the following hypotheses on $g$

$$
g \in C^{1}\left(\mathbb{R}^{+}\right) \cap L^{1}\left(\mathbb{R}^{+}\right), g(t)>0, \exists q_{0}, q_{1}>0:-q_{0} g(t) \leq g^{\prime}(t) \leq-q_{1} g(t), \forall t \geq 0 .
$$

In view of $(14)$, let $L_{g}^{2}\left(\mathbb{R}^{+} ; H_{0}^{1}(\Omega)\right)$ be the Hilbert space of $H_{0}^{1}(\Omega)$-value functions on $\mathbb{R}^{+}$, endowed with the inner product

$$
(f, h)_{L_{g}^{2}\left(\mathbb{R}^{+}, H_{0}^{1}(\Omega)\right)}=\int_{0}^{\infty} g(s) \int_{\Omega} \nabla f(x, s) \cdot \nabla \overline{h(x, s)} d x d s .
$$

To give an accurate formulation of the evolution problem we introduce the product Hilbert spaces

$$
\mathcal{H}=H_{0}^{1}(\Omega) \times L^{2}(\Omega) \times H_{0}^{1}(\Omega) \times L^{2}(\Omega) \times L_{g}^{2}\left(\mathbb{R}^{+} ; H_{0}^{1}(\Omega)\right)
$$


endowed with the following inner product

$$
\begin{aligned}
\langle U, V\rangle= & \beta_{0} \int_{\Omega} \nabla u_{1} \cdot \nabla \overline{v_{1}} d x+\int_{\Omega} u_{2} \overline{v_{2}} d x+\int_{\Omega} \nabla u_{3} \cdot \nabla \overline{v_{3}} d x+\int_{\Omega} u_{4} \overline{v_{4}} d x \\
& +\alpha \int_{\Omega}\left(u_{1} \overline{v_{3}}+u_{3} \overline{v_{1}}\right) d x+\int_{0}^{\infty} g(s) \int_{\Omega} \nabla u_{5}(x, s) \cdot \nabla \overline{v_{5}}(x, s) d x d s
\end{aligned}
$$

where $U=\left(u_{1}, u_{2}, u_{3}, u_{4}, u_{5}\right)^{T}, V=\left(v_{1}, v_{2}, v_{3}, v_{4}, v_{5}\right)^{T} \in \mathcal{H}$.

Let $U=\left(u, u_{t}, v, v_{t}, \eta\right)^{T}$ be and we define the operator $\mathcal{A}: D(\mathcal{A}) \subset \mathcal{H} \rightarrow \mathcal{H}$ given by

$$
\mathcal{A}=\left[\begin{array}{ccccc}
0 & I & 0 & 0 & 0 \\
\beta_{0} \Delta & 0 & -\alpha I & 0 & \mathcal{T} \\
0 & 0 & 0 & I & 0 \\
-\alpha I & 0 & \Delta & 0 & 0 \\
0 & I & 0 & 0 & -(\cdot)_{s}
\end{array}\right]
$$

with domain

$$
\begin{aligned}
D(\mathcal{A})= & \left\{(u, \varphi, v, \psi, \eta)^{T} \in \mathcal{H} ; \quad \beta_{0} u-\int_{0}^{\infty} g(s) \eta(s) d s \in H_{0}^{1}(\Omega) \cap H^{2}(\Omega),\right. \\
& \left.\varphi \in H_{0}^{1}(\Omega), v \in H_{0}^{1}(\Omega) \cap H^{2}(\Omega), \psi \in H_{0}^{1}(\Omega), \eta \in D(\mathcal{T})\right\}
\end{aligned}
$$

where

$$
\mathcal{T} \eta=\int_{0}^{\infty} g(s) \Delta \eta(s) d s, \quad \forall \eta \in D(\mathcal{T})
$$

with

$$
D(\mathcal{T})=\left\{\eta \in L_{g}^{2}\left(\mathbb{R}^{+} ; H_{0}^{1}(\Omega)\right) ; \eta_{s} \in L_{g}^{2}\left(\mathbb{R}^{+} ; H_{0}^{1}(\Omega)\right), \eta(0)=0\right\},
$$

where $\eta_{s}$ is the distributional derivative of $\eta$ with respect to the internal variable $s$. Therefore, the system (7)-(13) is equivalent to

$$
\begin{aligned}
\frac{d U}{d t} & =\mathcal{A} U \\
U(0) & =U_{0}
\end{aligned}
$$

with $U=\left(u, u_{t}, v, v_{t}, \eta\right)^{T}, U_{0}=\left(u_{0}, u_{1}, v_{0}, v_{1}, \eta_{0}\right)^{T}$. With the above notations, we have the following result.

Theorem 1. The operator $\mathcal{A}$ generate a $C_{0}$-semigroup $S(t)$ of contraction on $\mathcal{H}$. Thus, for any initial data $U_{0} \in \mathcal{H}$, the problem (7)-(13) has a unique weak solution $U(t) \in C^{0}\left(\left[0, \infty[, \mathcal{H})\right.\right.$. Moreover, if $U_{0} \in D(\mathcal{A})$, then $U(t)$ is strong solution of (7)-(13), that is, $U(t) \in C^{1}\left(\left[0, \infty[, \mathcal{H}) \cap C^{0}([0, \infty[, D(\mathcal{A}))\right.\right.$.

Proof. It is easy to see that $D(\mathcal{A})$ is dense in $\mathcal{H}$. Now, for $U=\left(u, u_{t}, v, v_{t}, \eta\right)^{T} \in D(\mathcal{A})$ and using the inner product (15), we get

$$
\begin{aligned}
& \langle\mathcal{A} U, U\rangle=\beta_{0} \int_{\Omega} \nabla u_{t} \cdot \nabla \bar{u} d x+\int_{\Omega}\left(\beta_{0} \Delta u-\alpha v+\int_{0}^{\infty} g(s) \Delta \eta(s) d s\right) \overline{u_{t}} d x \\
& \quad+\int_{\Omega} \nabla v_{t} \cdot \nabla \bar{v} d x+\int_{\Omega}(\Delta v-\alpha u) \overline{v_{t}} d x+\alpha \int_{\Omega}\left(u_{t} \bar{v}+v \overline{u_{t}}\right) d x+\int_{0}^{\infty} g(s) \int_{\Omega} \nabla\left(u_{t}-\eta_{s}(s)\right) \cdot \nabla \bar{\eta}(s) d x d s
\end{aligned}
$$

from where it follows that

$$
\langle\mathcal{A} U, U\rangle=-\int_{0}^{\infty} g(s) \int_{\Omega} \nabla \eta_{s}(s) \cdot \nabla \bar{\eta}(s) d x d s .
$$

Integrating by parts and using (14), we have

$$
\mathcal{R} e\langle\mathcal{A} U, U\rangle=\frac{1}{2} \int_{0}^{\infty} g^{\prime}(s) \int_{\Omega}|\nabla \eta(s)|^{2} d x d s \leq-\frac{q_{1}}{2} \int_{0}^{\infty} g(s) \int_{\Omega}|\nabla \eta(s)|^{2} d x d s \leq 0 .
$$


Therefore, $\mathcal{A}$ is a dissipative operator.

Next, we show that $(I-\mathcal{A})$ is maximal. For this, let us consider the equation

$$
(I-\mathcal{A}) U=F
$$

where $U=(u, \varphi, v, \psi, \eta)^{T}$ and $F=\left(f^{1}, f^{2}, f^{3}, f^{4}, f^{5}\right)^{T} \in \mathcal{H}$. Then, in terms of its components, we can write

$$
\begin{aligned}
u-\varphi & =f^{1}, \\
\varphi-\beta_{0} \Delta u+\alpha v-\int_{0}^{\infty} g(s) \Delta \eta(s) d s & =f^{2}, \\
v-\psi & =f^{3}, \\
\psi-\Delta v+\alpha u & =f^{4}, \\
\eta-\varphi+\eta_{s} & =f^{5} .
\end{aligned}
$$

Integrating (22), we have

$$
\eta(\cdot, s)=\varphi(\cdot)\left(1-e^{-s}\right)+\int_{0}^{s} e^{\tau-s} f^{5}(\cdot, \tau) d \tau .
$$

Substituting $\varphi$ and $\eta$ from (18) and (23) into (19), we get

$$
u-\beta_{g} \Delta u+\alpha v=f^{1}+f^{2}+\int_{0}^{\infty} g(s)\left[\left(e^{-s}-1\right) \Delta f^{1}+\int_{0}^{s} e^{\tau-s} \Delta f^{5}(\tau) d \tau\right] d s
$$

where

$$
\beta_{g}=\beta_{0}+\int_{0}^{\infty} g(s)\left(1-e^{-s}\right) d s
$$

Note that $\beta_{g}$ is a positive constant in virtue of (14). Moreover, it can be shown that the right-had side of (24) is in $H^{-1}(\Omega)$.

On the other hand, the substitution of $\psi$ given in (20) into (21) gives us

$$
v-\Delta v+\alpha u=f^{3}+f^{4} .
$$

First we prove that $u, v \in H_{0}^{1}(\Omega)$. To do this, let us consider the bilinear form

$$
\begin{aligned}
a\left(\Phi_{1}, \Phi_{2}\right) & =\int_{\Omega} u_{1} u_{2} d x+\int_{\Omega} v_{1} v_{2} d x+\beta_{g} \int_{\Omega} \nabla u_{1} \cdot \nabla u_{2} d x \\
& +\int_{\Omega} \nabla v_{1} \cdot \nabla v_{2} d x+\alpha \int_{\Omega}\left(v_{1} u_{2}+u_{1} v_{2}\right)
\end{aligned}
$$

where $\Phi_{1}=\left(u_{1}, v_{1}\right)$ and $\Phi_{2}=\left(u_{2}, v_{2}\right)$.

Then, Lax-Milgram theorem (see [19]) provides existence and uniqueness of the solutions

$$
u, v \in H_{0}^{1}(\Omega) .
$$

From (18) and (20), we have $\varphi, \psi \in H_{0}^{1}(\Omega)$.

Now, from (23), we obtain

$$
\|\eta\|_{L_{\delta}^{2}\left(\mathbb{R}^{+} ; H_{0}^{1}(\Omega)\right)}^{2} \leq C\left(\|\varphi\|_{H_{0}^{1}(\Omega)}^{2}+\left\|f^{5}\right\|_{L_{S}^{2}\left(\mathbb{R}^{+} ; H_{0}^{1}(\Omega)\right)}^{2}\right),
$$

from where it follows that

$$
\eta \in L_{g}^{2}\left(\mathbb{R}^{+} ; H_{0}^{1}(\Omega)\right)
$$

From (19), we get

$$
\beta_{0} \Delta u+\int_{0}^{\infty} g(s) \Delta \eta(s) d s \in L^{2}(\Omega)
$$


On the other hand, from (22), we obtain

$$
\left\|\eta_{s}\right\|_{L_{g}^{2}\left(\mathbb{R}^{+} ; H_{0}^{1}(\Omega)\right)}^{2} \leq C\left(\|\varphi\|_{H_{0}^{1}(\Omega)}^{2}+\left\|f^{5}\right\|_{L_{g}^{2}\left(\mathbb{R}^{+} ; H_{0}^{1}(\Omega)\right)}^{2}+\|\eta\|_{L_{g}^{2}\left(\mathbb{R}^{+} ; H_{0}^{1}(\Omega)\right)}^{2}\right) .
$$

From where it follows that

$$
\eta_{s} \in L_{g}^{2}\left(\mathbb{R}^{+} ; H_{0}^{1}(\Omega)\right)
$$

Again from (23), we have

$$
\eta(0)=0 .
$$

Thus, $I-\mathcal{A}$ is maximal. Then, thanks to the Lumer-Phillips theorem (see [13], Theorem 4.3), the operator $\mathcal{A}$ generates a $\mathrm{C}_{0}$-semigroup of contractions $e^{t \mathcal{A}}$ on $\mathcal{H}$. The proof is now complete.

\section{Lack of exponential decay}

Our starting point is to show that the semigroup associated to the system (7)-(13) is not exponential stable. To show this, we assume that $g(t)=e^{-\mu t}$, with $t \in \mathbb{R}^{+}$and $\mu>1$. We will use the Prüss's theorem [14] to prove the lack of exponential stability.

Theorem 2. Let $S(t)=e^{\mathcal{A t}}$ be a $C_{0}$-semigroup of contractions on Hilbert space. Then $S(t)$ is exponentially stable if and only if

$$
\rho(\mathcal{A}) \supseteq\{i \beta: \beta \in \mathbb{R}\} \equiv i \mathbb{R}
$$

and

$$
\varlimsup_{|\beta| \rightarrow \infty}\left\|(i \beta-\mathcal{A})^{-1}\right\|<\infty
$$

hold, where $\rho(\mathcal{A})$ is the resolvent set of $\mathcal{A}$.

To do this, let us consider the spectral problem:

$$
\left\{\begin{aligned}
&-\Delta w_{m}=\lambda_{m} w_{m} \text { in } \quad \Omega \\
& w_{m}=0 \text { on } \quad \Gamma,
\end{aligned}\right.
$$

where

$$
\lim _{m \rightarrow \infty} \lambda_{m}=+\infty .
$$

The following theorem describes the main results of this section.

Theorem 3. Let $S(t)$ be $C_{0}$-semigroup of contractions generated by $\mathcal{A}$. Then $S(t)$ is not exponentially stable.

Proof. Here we will use the Theorem 2. That is, we will show that there exists a sequence of values $\lambda_{m}$ such that

$$
\left\|\left(\lambda_{m}-\mathcal{A}\right)^{-1}\right\|_{\mathcal{L}(\mathcal{H})} \rightarrow \infty .
$$

It is equivalent to prove that there exist a sequence of data $F_{m} \in \mathcal{H}$ and a sequence of complex numbers $\lambda_{m} \in i \mathbb{R}$, with $\left\|F_{m}\right\|_{\mathcal{H}} \leq 1$ such that

$$
\left\|\left(\lambda_{m} I-\mathcal{A}\right)^{-1} F_{m}\right\|_{\mathcal{H}} \rightarrow \infty
$$

where

$$
\lambda_{m} U_{m}-\mathcal{A} U_{m}=F_{m}
$$

with $U_{m}$ not bounded.

To simplify the notation we will omit the subindex $m$. Then, the Equation (31) becomes 


$$
\left\{\begin{array}{c}
i \lambda u-\varphi=f^{1} \\
i \lambda \varphi-\beta_{0} \Delta u+\alpha v-\int_{0}^{\infty} g(s) \Delta \eta(x, s) d s=f^{2} \\
i \lambda v-\psi=f^{3} \\
i \lambda \psi-\Delta v+\alpha u=f^{4} \\
i \lambda \eta-\varphi+\eta_{s}=f^{5} .
\end{array}\right.
$$

Let us consider $f 1=f^{3}=f^{5}=0$ and $f^{2}=f^{4}=w_{m}$ to obtain $\varphi=i \lambda u$ e $\psi=i \lambda v$. Then, the system (32) becomes

$$
\left\{\begin{array}{c}
-\lambda^{2} u-\beta_{0} \Delta u+\alpha v-\int_{0}^{\infty} g(s) \Delta \eta(x, s) d s=w_{m} \\
-\lambda^{2} v-\Delta v+\alpha u=w_{m} \\
i \lambda \eta+\eta_{s}-i \lambda u=0 .
\end{array}\right.
$$

We look for solutions of the form

$$
u=a w_{m}, \quad v=b w_{m}, \quad \varphi=c w_{m}, \quad \psi=d w_{m}, \quad \eta(x, s)=\gamma(s) w_{m}
$$

with $a, b, c, d \in \mathbb{C}$ and $\gamma(s)$ depend on $\lambda$ and will be determined explicitly in the sequel. From (33), we get $a$ and $b$ satisfy

$$
\left\{\begin{array}{c}
-\lambda^{2} a+\beta_{0} a \lambda_{m}+\alpha b+\lambda_{m} \int_{0}^{\infty} g(s) \gamma(s) d s=1 \\
-\lambda^{2} b+\lambda_{m} b+\alpha a=1 \\
\gamma_{s}+i \lambda \gamma-i \lambda a=0
\end{array}\right.
$$

Solving $(34)_{3}$ we get

$$
\gamma(s)=C e^{-i \lambda s}+a .
$$

Since $\eta(0)=0$ then $C=-a$, and (35) becomes

$$
\gamma(s)=a-a e^{-i \lambda s} .
$$

Then, from (36) we have

$$
\int_{0}^{\infty} g(s) \gamma(s) d s=\int_{0}^{\infty} g(s)\left(a-a e^{-i \lambda s}\right) d s=a b_{0}-a \int_{0}^{\infty} g(s) e^{-i \lambda s} d s
$$

where

$$
b_{0}=\int_{0}^{\infty} g(s) d s .
$$

Now, choosing $\lambda=\sqrt{\lambda_{m}}$, using the equation (34) $)_{1}$ and (34) $)_{2}$ we obtain

$$
\begin{aligned}
& a=\frac{1}{\alpha} \\
& b=\frac{\lambda_{m}\left(1-\beta_{0}\right)}{\alpha^{2}}-\frac{\lambda_{m}}{\alpha} \int_{0}^{\infty} g(s) \gamma(s) d s+\frac{1}{\alpha}, \\
& c=i \frac{\sqrt{\lambda_{m}}}{\alpha}, \\
& d=i \sqrt{\lambda_{m}}\left(\frac{\lambda_{m}\left(1-\beta_{0}\right)}{\alpha^{2}}-\frac{\lambda_{m}}{\alpha} \int_{0}^{\infty} g(s) \gamma(s) d s+\frac{1}{\alpha}\right) .
\end{aligned}
$$

Recalling that

$$
\varphi=c w_{m}=i \frac{\sqrt{\lambda_{m}}}{\alpha} w_{m}
$$


we get

$$
\|\varphi\|_{L^{2}(\Omega)}^{2}=\frac{\lambda_{m}}{\alpha^{2}}
$$

Therefore we have

$$
\lim _{m \rightarrow \infty}\left\|U_{m}\right\|_{\mathcal{H}}^{2} \geq \lim _{m \rightarrow \infty}\|\varphi\|_{L^{2}(\Omega)}^{2}=\lim _{m \rightarrow \infty} \frac{\lambda_{m}}{\alpha^{2}}=\infty .
$$

Using theorem 3 follows that $S(t)$ is not exponentially stable. The proof is now complete.

\section{Polynomial decay and optimally result}

In this section we study the polynomial decay associated to the system (7)-(13) and subsequently we find the optimal rate of decay. Then, let us consider the resolvent equation

$$
(i \lambda I-\mathcal{A}) U=F, \quad \text { with } \quad \lambda \in \mathbb{R} \text { and } F \in \mathcal{H},
$$

that is,

$$
\begin{aligned}
i \lambda u-\varphi & =f^{1}, \\
i \lambda \varphi-\beta_{0} \Delta u+\alpha v-\mathcal{T} \eta & =f^{2}, \\
i \lambda v-\psi & =f^{3}, \\
i \lambda \psi-\Delta v+\alpha u & =f^{4}, \\
i \lambda \eta-\varphi+\eta_{s} & =f^{5} .
\end{aligned}
$$

In the next step we shall show three lemmas important to proof the main result.

Lemma 1. The solutions of the system (7)-(13), given by the Theorem 1, satisfies

$$
\int_{\Omega} \int_{0}^{\infty} g(s)|\nabla \eta|^{2} d s d x \leq K|\lambda|^{2}|| U\left\|_{\mathcal{H}}\right\| F \|_{\mathcal{H}}
$$

where $K$ is a positive constant and $|\lambda|>1$.

Proof. Multiplying the equality (39) by $\bar{\varphi}$ and integrating by parts on $\Omega$, we get

$$
i \lambda \int_{\Omega}|\varphi|^{2} d x+\underbrace{\beta_{0} \int_{\Omega} \nabla u \cdot \nabla \bar{\varphi} d x}_{:=I_{1}}+\underbrace{\alpha \int_{\Omega} v \bar{\varphi} d x}_{:=I_{2}}+\underbrace{\int_{\Omega} \int_{0}^{\infty} g(s) \nabla \eta(s) \cdot \nabla \bar{\varphi} d s d x}_{:=I_{3}}=\int_{\Omega} f^{2} \bar{\varphi} d x .
$$

Substituting $\varphi$ given in (38) into $I_{1}$ and $I_{2}$, we have

$$
I_{1}=-i \lambda \beta_{0} \int_{\Omega}|\nabla u|^{2} d x-\beta_{0} \int_{\Omega} \nabla u \cdot \nabla \overline{f^{1}} d x
$$

and

$$
I_{2}=-i \lambda \alpha \int_{\Omega}|u|^{2} d x-\alpha \int_{\Omega} u \overline{f^{1}} d x
$$

Now, substituting $\varphi$ given in (42) into $I_{3}$ and integrating by parts, we obtain

$$
I_{3}=-i \lambda \int_{\Omega} \int_{0}^{\infty} g(s)|\nabla \eta(s)|^{2} d s d x-\int_{\Omega} \int_{0}^{\infty} g^{\prime}(s)|\nabla \eta(s)|^{2} d s d x-\int_{\Omega} \int_{0}^{\infty} g(s) \nabla \eta(s) \cdot \nabla \overline{f^{5}} d s d x d x .
$$

Substituting (44), (45) and (46) into (43), we get

$$
\begin{aligned}
& i \lambda \int_{\Omega}|\varphi|^{2} d x-i \lambda \beta_{0} \int_{\Omega}|\nabla u|^{2} d x-i \lambda \alpha \int_{\Omega}|u|^{2} d x-i \lambda \int_{\Omega} \int_{0}^{\infty} g(s)|\nabla \eta(s)|^{2} d s d x-\frac{1}{2} \int_{\Omega} \int_{0}^{\infty} g^{\prime}(s)|\nabla \eta(s)|^{2} d s d x \\
& =\beta_{0} \int_{\Omega} \nabla u \cdot \nabla \overline{f^{1}} d x+\alpha \int_{\Omega} u \overline{f^{1}} d x+\int_{\Omega} \int_{0}^{\infty} g(s) \nabla \eta(s) \cdot \nabla \overline{f^{5}} d s d x+\int_{\Omega} f^{2} \bar{\varphi} d x
\end{aligned}
$$


Taking the real part on the left side of the above equality and using the hypotheses (14) on $g$, our conclusion follows.

Lemma 2. For any $\epsilon>0$, there exists a positive constant $K_{\epsilon}$ such that

$$
\begin{aligned}
& \beta_{0} \int_{\Omega}|\nabla u|^{2} d x+\int_{\Omega}|\nabla v|^{2} d x+\alpha \int_{\Omega}(u \bar{v}+v \bar{u}) d x \\
& \leq \int_{\Omega}|\varphi|^{2} d x+\int_{\Omega}|\psi|^{2} d x+\epsilon \int_{\Omega}|\nabla u|^{2} d x+\left.\left.K_{\epsilon}|\lambda|^{2}|| U\right|_{\mathcal{H}}|| F\right|_{\mathcal{H}}+K|| U\left\|_{\mathcal{H}}|| F\right\|_{\mathcal{H}}
\end{aligned}
$$

where $K$ is a positive constant.

Proof. Multiplying the equalities (39) and (41) by $\bar{u}$ and $\bar{v}$, respectively, integrating by parts on $\Omega$ and summing up the result, we get

$$
\begin{aligned}
& \underbrace{i \lambda \int_{\Omega} \varphi \bar{u} d x}_{:=I_{4}}+\beta_{0} \int_{\Omega}|\nabla u|^{2} d x+\alpha \int_{\Omega} v \bar{u} d x+\int_{\Omega} \int_{0}^{\infty} g(s) \nabla \eta(s) \cdot \nabla \bar{u} d s d x \\
& +\underbrace{i \lambda \int_{\Omega} \psi \bar{v} d x}_{:=I_{5}}+\int_{\Omega}|\nabla v|^{2} d x+\alpha \int_{\Omega} u \bar{v} d x=\int_{\Omega} f^{2} \bar{u} d x+\int_{\Omega} f^{4} \bar{v} d x .
\end{aligned}
$$

Substituting $\overline{i \lambda u}$ given in (38) into $I_{4}$ and $\overline{i \lambda v}$ given in (40) into $I_{5}$, we find

$$
\begin{aligned}
& \beta_{0} \int_{\Omega}|\nabla u|^{2} d x+\int_{\Omega}|\nabla v|^{2} d x+\alpha \int_{\Omega}(u \bar{v}+v \bar{u}) d x=\int_{\Omega}\left(|\varphi|^{2}+|\psi|^{2}\right) d x-\int_{0}^{\infty} g(s) \int_{\Omega} \nabla \eta \cdot \nabla \bar{u} d x \\
& +\int_{\Omega} \varphi \overline{f^{1}} d x+\int_{\Omega} f^{2} \bar{u} d x+\int_{\Omega} \psi \overline{f^{3}} d x+\int_{\Omega} f^{4} \bar{v} d x .
\end{aligned}
$$

Now, using Poincaré and Young inequalities, we have

$$
\begin{aligned}
& \beta_{0} \int_{\Omega}|\nabla u|^{2} d x+\int_{\Omega}|\nabla v|^{2} d x+\alpha \int_{\Omega}(u \bar{v}+v \bar{u}) d x \\
& \leq \int_{\Omega}\left(|\varphi|^{2}+|\psi|^{2}\right) d x+\epsilon \int_{\Omega}|\nabla u|^{2} d x+K_{\epsilon}\|\eta\|_{L_{\bar{g}}^{2}\left(\mathbb{R}^{+} ; H_{0}^{1}(\Omega)\right)}^{2}+K\|U\|_{\mathcal{H}}\|F\|_{\mathcal{H}} .
\end{aligned}
$$

Using the Lemma 1, our conclusion follows.

Lemma 3. Under the conditions of the previous lemma, we have

$$
\frac{b_{0}}{2} \int_{\Omega}|\varphi|^{2} d x \leq \epsilon \int_{\Omega}\left(|\nabla u|^{2}+|\nabla v|^{2}\right) d x+K_{\epsilon}|\lambda|^{2}|| U\left\|_{\mathcal{H}}|| F\right\|_{\mathcal{H}}+K\|U\|_{\mathcal{H}}\|F\|_{\mathcal{H}}
$$

and

$$
\left(\frac{1}{2}-\frac{K}{|\lambda|^{2}}\right) \int_{\Omega}|\psi|^{2} d x \leq K_{\epsilon}|\lambda|^{2}|| U\left\|_{\mathcal{H}}\right\| F\left\|_{\mathcal{H}}+K\right\| U\left\|_{\mathcal{H}}\right\| F \|_{\mathcal{H}}
$$

with $|\lambda|>1$ large enough.

Proof. Multiplying the equation (42) by $\int_{0}^{\infty} g(s) d s \bar{\varphi}$ and integrating on $\Omega$, we find

$$
\underbrace{i \lambda \int_{0}^{\infty} g(s) \int_{\Omega} \eta(s) \bar{\varphi} d x d s}_{:=I_{6}}-b_{0} \int_{\Omega}|\varphi|^{2} d x+\int_{0}^{\infty} g(s) \int_{\Omega} \eta_{s}(s) \bar{\varphi} d x d s=\int_{0}^{\infty} g(s) \int_{0}^{\infty} f^{5}(s) \bar{\varphi} d x d s
$$

where $b_{0}=\int_{0}^{\infty} g(s) d s$. On the other hand, noting that

$$
\int_{0}^{\infty} g(s) \int_{\Omega} \eta_{s}(s) \bar{\varphi} d x d s=-\int_{0}^{\infty} g^{\prime}(s) \int_{\Omega} \eta(s) \bar{\varphi} d x d s
$$


and substituting $\overline{i \lambda \varphi}$ given in (39) into $I_{6}$, we get

$$
\begin{aligned}
& b_{0} \int_{\Omega}|\varphi|^{2} d x=-\beta_{0} \int_{0}^{\infty} g(s) \int_{\Omega} \eta(s) \Delta \bar{u} d x d s+\alpha \int_{0}^{\infty} g(s) \int_{\Omega} \eta(s) \bar{v} d x d s-\alpha \int_{\Omega}\left(\int_{0}^{\infty} g(s) \eta(s) d s\right) \\
& \times\left(\int_{0}^{\infty} g(s) \Delta \eta(s) d s\right)+\int_{0}^{\infty} g^{\prime}(s) \int_{\Omega} \eta(s) \bar{\varphi} d x d s-\int_{0}^{\infty} g(s) \int_{\Omega} \eta(s) \overline{f^{2}} d x d s
\end{aligned}
$$
have

Using the hypotheses on $g$ given in (14) and taking into account the Poincaré and Young inequalities, we

$$
\frac{b_{0}}{2} \int_{\Omega}|\varphi|^{2} d x \leq \epsilon \int_{\Omega}\left(|\nabla u|^{2}+|\nabla v|^{2}\right) d x+K_{\epsilon}\|\eta\|_{L_{g}^{2}\left(\mathbb{R}^{+} ; H_{0}^{1}(\Omega)\right)}^{2}+K\|U\|_{\mathcal{H}}\|F\|_{\mathcal{H}}
$$

Using the Lemma 1, follows the first inequality.

To show the second inequality, we substitute the equation (38) into (42). This gives,

$$
i \lambda \eta-i \lambda u+\eta_{s}=f^{5}-f^{1} .
$$

Now, we substitute $u$ given in (41) into (52). Then, we obtain,

$$
i \lambda \alpha \eta-\lambda^{2} \psi-i \lambda \Delta v+\alpha \eta_{s}=\alpha\left(f^{5}-f^{1}\right)+i \lambda f^{4}
$$

Multiplying the equation (53) by $\overline{\int^{\infty} g(s) \psi}$, integrating by parts on $\Omega$ and proceeding as to obtain the first estimate, we have

$$
\frac{1}{2} \int_{\Omega}|\psi|^{2} d x \leq \frac{K}{|\lambda|^{2}} \int_{\Omega}|\psi|^{2} d x+K|\lambda|^{2}|| U\left\|_{\mathcal{H}}|| F\right\|_{\mathcal{H}}+\left.K|| F\right|_{\mathcal{H}} ^{2}
$$

From where follows the second inequality. The proof is now complete.

Now, we are in the position the main result of this paper.

Theorem 4. The semigroup associated to the system (7)-(13) is polynomially stable and

$$
\left\|S(t) U_{0}\right\|_{\mathcal{H}} \leq \frac{K}{\sqrt{t}}\left\|U_{0}\right\|_{D(\mathcal{A})} .
$$

Moreover, this result is optimal.

Proof. From Lemmas 1, 2 and 3, choosing $\epsilon>0$ small enough and for $|\lambda|>1$ large enough, we have

$$
\|U\|_{\mathcal{H}}^{2} \leq K|\lambda|^{2}|| U\left\|_{\mathcal{H}}\right\| F\left\|_{\mathcal{H}}+K|| F\right\|_{\mathcal{H}}^{2} .
$$

From where it follows that

$$
\|U\|_{\mathcal{H}}^{2} \leq\left. K|\lambda|^{4}|| F\right|_{\mathcal{H}} ^{2}
$$

that can be written as

$$
\|(\lambda I-\mathcal{A})^{-1}|| \leq K|\lambda|^{2}
$$

that is

$$
\left\|(\lambda I-\mathcal{A})^{-1}\right\|=K \mathcal{O}\left(|\lambda|^{2}\right), \lambda \rightarrow \infty .
$$

Then using the Theorem of A. Borichev and Y. Tomilov, (see [15], Theorem 2.4), the condition (54) is equivalent to

$$
\left\|S(t) \mathcal{A}^{-1}\right\|=K \mathcal{O}\left(t^{-\frac{1}{2}}\right) \Rightarrow\left\|S(t) \mathcal{A}^{-1} F\right\|_{\mathcal{H}} \leq \frac{K}{\sqrt{t}}\|F\|_{\mathcal{H}}, t \rightarrow \infty .
$$


Then taking $\mathcal{A} U_{0}=F$, we get

$$
\left\|S(t) U_{0}\right\|_{\mathcal{H}} \leq \frac{K}{\sqrt{t}}\left\|U_{0}\right\|_{D(\mathcal{A})} .
$$

Therefore the solution decays polynomially.

To prove that the rate of decay is optimal, we will argue by contradiction. Suppose that the rate $t^{-\frac{1}{2}}$ can be improved; for example that the rate is $t^{-\frac{1}{2-\epsilon}}$ for some $0<\epsilon<2$. From Theorem 5.3 in [20], the operator

$$
|\lambda|^{-2+\frac{\epsilon}{2}}\left\|(\lambda I-\mathcal{A})^{-1}\right\|
$$

should be limited, but this does not happen. For this, let us suppose that there exist a sequence $\left(\lambda_{\mu}\right) \subset \mathbb{R}$ with $\lim _{\mu \rightarrow \infty}\left|\lambda_{\mu}\right|=\infty$ and $\left(U_{\mu}\right) \subset D(\mathcal{A})$ for $\left(F_{\mu}\right) \subset \mathcal{H}$ such that

$$
\left(i \lambda_{\mu} I-\mathcal{A}\right) U_{\mu}=F_{\mu}
$$

is bounded in $\mathcal{H}$ and

$$
\lim _{\mu \rightarrow \infty}\left|\lambda_{\mu}\right|^{-2+\frac{\epsilon}{2}}\left\|U_{\mu}\right\|_{\mathcal{H}}=\infty .
$$

So, following the same steps as in the proof of Theorem 3 we can conclude that

$$
\left|\lambda_{\mu}\right|^{-2+\frac{\epsilon}{2}}\left\|U_{\mu}\right\|_{\mathcal{H}} \geq \mathcal{O}\left(\mu^{\frac{\epsilon}{2}}\right) \rightarrow \infty, \quad \text { as } \mu \rightarrow \infty .
$$

Therefore the rate cannot be improved. The proof is now complete.

Acknowledgments: This research is partially supported by PNPD/UFBA/CAPES(Brazil).

Author Contributions: All authors contributed equally to the writing of this paper. All authors read and approved the final manuscript.

Conflicts of Interest: "The authors declare no conflict of interest."

\section{References}

[1] Raposo, C. A., Bastos, W. D., \& Alves, B. F. (2010). Loss of exponential stability for a thermoelastic system with memory. Electronic Journal of Differential Equations, 15, 1-5.

[2] Cannon, J. R. (1963). The solution of heat equation subject to the specification of energy. Quarterly of Applied Mathematics, 21, 155-160.

[3] Bažant, Z. P., \& Jirásek, M. (2002). Nonlocal integral formulation of plasticity and damage: Survey of progress. Journal of Engineering Mechanics, 128, 1119-1149.

[4] Komornik, V., \& Bopeng, R. (1997). Boundary stabilization of compactly coupled wave equations. Asymptotic Analysis, 14, 339-359.

[5] Aassila, M. (1999). A note on the boundary stabilization of a compactly coupled system of wave equations. Applied Mathematics Letters, 12, 19-24.

[6] Aassila, M. (2001). Strong asymptotic stability of a compactly coupled system of wave equations, Applied Mathematics Letters, 14, 285-290.

[7] Bastos, W. D., Spezamiglio, A., \& Raposo, C. A. (2011). On exact boundary controllability for linearly coupled wave equations. Journal of Mathematical Analysis and Applications, Article.ID 15692.

[8] Najafi, M. (2001). Study of exponential stability of coupled wave systems via distributed stabilizer. International Journal of Mathematics and Mathematical Sciences, 28, 479-491.

[9] Lobato, R. F. C., Cordeiro, S. M. C., Santos, M. L., \& Almeida Junior, D. S. (2014). Optimal polynomial decay to coupled wave equations and its numerical properties. Journal of Applied Mathematics., Art. ID 897080.

[10] Boussoira, F. A. (1999). Stabilisation frontière indirecte de systèmes faiblement couplés. Comptes rendus de l'Académie des Sciences, 328, 1015-1020.

[11] Boussoira, F. A., Cannarsa, P., \& Komornik, V. (2002). Indirect internal stabilization of weakly coupled evolution equations. Journal of Evolution Equations, 2, 127-150. 
[12] Almeida, R. G. C., \& Santos, M. L. (2011). Lack of exponential decay of a coupled system of wave equations with memory. Nonlinear Analysis: Real World Applications, 12, 1023-1032.

[13] Pazy, A. (1983). Semigroups of linear operators and applications to partial differential equations. Springer-Verlag, New York.

[14] Prüss, J. (1984). On the spectrum of $\mathrm{C}_{0}$-semigroups. Transactions of the American Mathematical Society, $28,847-857$.

[15] Borichev, A., \& Tomilov, Y. (2009). Optimal polynomial decay of functions and operator semigroups. Mathematische Annalen, 347, 455-478.

[16] Dafermos, C. M. (1970). Asymptotic stability in viscoelasticity. Archive for Rational Mechanics and Analysis, 37, $297-308$.

[17] Fabrizio, M., \& Morro, A. (1992). Mathematical problems in linear viscoelasticity. SIAM Studies in Applied Mathematics, Philadelphia.

[18] Rivera, J. E. M., \& Naso, M. G. (2007). Asymptotic stability of semigroups associated with linear weak dissipative systems with memory. Journal of Mathematical Analysis and Applications, 326, 691-707.

[19] Brezis, H. (1992). Analyse Fonctionelle, Théorie et Applications. Masson, Paris.

[20] Fatori, L. H., \& Rivera, J. E. M. (2010). Rates of decay to weak thermoelastic Bresse system. IMA Journal of Applied Mathematics, 75, 881-904.

(c) 2020 by the authors; licensee PSRP, Lahore, Pakistan. This article is an open access article distributed under the terms and conditions of the Creative Commons Attribution (CC-BY) license (http://creativecommons.org/licenses/by/4.0/). 